(Aus dem physiologischen Institut der Universität Halle a./S.)

\title{
Ueber den Einfluss der Spannungszunahme während der Zuckung auf die Arbeitsleistung des Muskels und den Verlauf der Curve.
}

Von

Erich Meyer, cand. med.

(Mit 2 Textfiguren und 2 Tafeln.)

Der Einfluss der Spannung auf die Muskelthätigkeit ist vielfach Gegenstand der Untersuchung gewesen. Nachdem man erfahren, dass die Arbeitsleistung und Wärmeentwicklung im Muskel mit zunehmender Anfangsspannung; d. h. mit zunehmender Belastung v o $r$ der Reizung wächst, lag die Frage nahe, ob auch eine Spannungsänderung nach der Reizung Einfluss auf die Muskelthätigkeit habe. Zur Beantwortung dieser Frage sind eine Reihe von Versuchen angestellt worden, die man alle mit dem gemeinsamen Namen Versuche über "Wechselzuckungen" belegen kann, ein Ausdruck, der von v. Kries ${ }^{1}$ ) schon zusammenfassend für seine Anschlags- und die von Helmholtz eingeführten Ueberlastungszuckungen gebraucht wurde. Aber Wechselzuckungen sind auch die Fick' $\operatorname{schen}^{2}$ ) Zuckungen mit Anfangshemmung, die Wurfzuckungen; zu ihnen gehören ferner die Schleuderzuckungen, Zugzuckungen und Zugschleuderzuckungen Schenck's ${ }^{3}$ ). Denn bei diesen allen findet nach der Reizung im Gegensatz zur isotonischen Zuckung ein Spannungswechsel statt. -

1) v. Kries, Untersuchungen zur Mechanik des quergestreiften Muskels. Du Bois' Archiv 1892.

2) A. Fick, Myothermische Fragen und Versuche (Verhandl. der physikalischmedicinischen Gesellschaft zu Würzburg.

3) Fr. Schenk, Pflüger's Archiv Bd. 51 S. 509 (1892), Einfluss der Spannung auf die Wärmebildung des Muskels und Bd. 61 S. 77 f. (1895), Weitere Untersuchungen über den Einfluss der Spannung auf den Zuckungsverlauf. 
Aus den Untersuchungen von Helmboltz wissen wir, wie dieser Spannungswechsel auf die Arbeitsleistung des Muskels wirkt, wenn er gleich zu Beginn der Contraction erfolgt. Wachsende Ueberlastung steigert die Arbeitsleistung des Muskels geradeso wie wachsende Anfangsbelastung. Dementsprechend fand Heidenhain ${ }^{1}$ ) durch thermische Versuche, dass mit zunehmender Ueberlastung auch die Wärmebildung bis zu einem Maximum steigt. Diese sehr zweckmässige Anpassung an verschieden grosse Anforderungen legt die Frage nahe, ob auch während der Zuckung selbst in einem beliebigen Momente derselben der Muskel dieses Anpassungsvermögen besitzt; d. h. wie verhält sich der Muskel, wenn in einem beliebigen Momente der Zuckung seine Last vermehrt wird? Vermag er dann eine grössere Arbeit zu leisten, als bei einfach isotonischer Zuckung, wenn in beiden Fällen dieselbe Anfangsspannung herrseht, und wächst die Arbeitsleistung bei zunehmender zweiter Belastung? Eine solche Zuckung, die eben auch zu den Wechselzuckungen gehört, nenne ich im Folgenden nach Professor Bernstein's Vorschlag im Gegensatz zu der isotonischen oder monotonischen eine ditonische ${ }^{2}$ ). Es handelt sich also bei unseren Versuchen, die ich auf Veranlassung und unter Leitung von Professor Bernstein unternahm, darum, die Arbeitsleistung des Muskels bei ditonischer Zuckung zu messen. Hierzu wurde folgende einfache Vorrichtung benützt: An einer Säule (Fig. 1) befinden sich senkrecht unter einander zwei ganz gleiche Aluminiumschreibhebel. Beide werden mittels einer dünnen Seidenschnur mit einander verbunden. Der obere Hebel wird nahe seiner Achse mittels einer Rolle in gewöhnlicher Weise isotonisch nach Fick belastet $\left(p_{1}\right)$. An den unteren Hebel wird das während der Contraction zu hebende zweite Gewicht $\left(p_{2}\right)$ angehängt. Das Muskelpräparat (Semimembranosus und Gracilis grosser Frösche) wurde an dem Unterschenkelknochen aufgehängt und an dem Beckenende mittels eines Häkchens mit dem oberen Schreibhebel verbunden. Der Angriffspunkt des Muskels und der des zweiten Gewichts $\left(p_{2}\right)$ fallen,

1) R. Heidenhain, Mechanische Leistung, Wärmeentwicklung und Stoffumsatz bei der Muskelthätigkeit S. 103. Leipzig 1864. Breitkopf \& Härtel.

2) v. Kries hat gelegentlich anch Zuckungen mit Lastvermehrung gezeichnet mit Hülfe einer etwas complicirten Einrichtung, bei welcher ein Elektromagnet die Last vermehren oder vermindern (Entlastungszuckungen) konnte (D u B o is' Archiv 1880, Untersuchungen zur Mechanik des quergestreiften Muskels). Doch sind die im Folgenden behandelten Fragen hierbei nicht berücksichtigt. 
Ueb. d. Einfluss d. Spannungszunahme während d. Zuckung a. d. Arbeitsl. etc. 595

wie aus der Figur ersichtlich, zusammen ${ }^{1}$ ). An einem Metallarm befindet sich nach oben und unten verschieblich ein horizontal und senkrecht zur Ebene der beiden Hebel gestellter Metallstab, dessen Querschnitt $A$ in der Figur sichtbar ist. Dieser dieser dient dem unteren Hebel als Unterlage. Schiebt man den Stab mit dem darauf ruhenden Hebel so weit herab, dass der Seidenfaden zwischen den beiden Hebeln straff gespannt ist, so werden beide Hebel und die an ihnen befestigten Lasten zu gleicher Zeit vom Muskel gehoben.

Fig. 1.

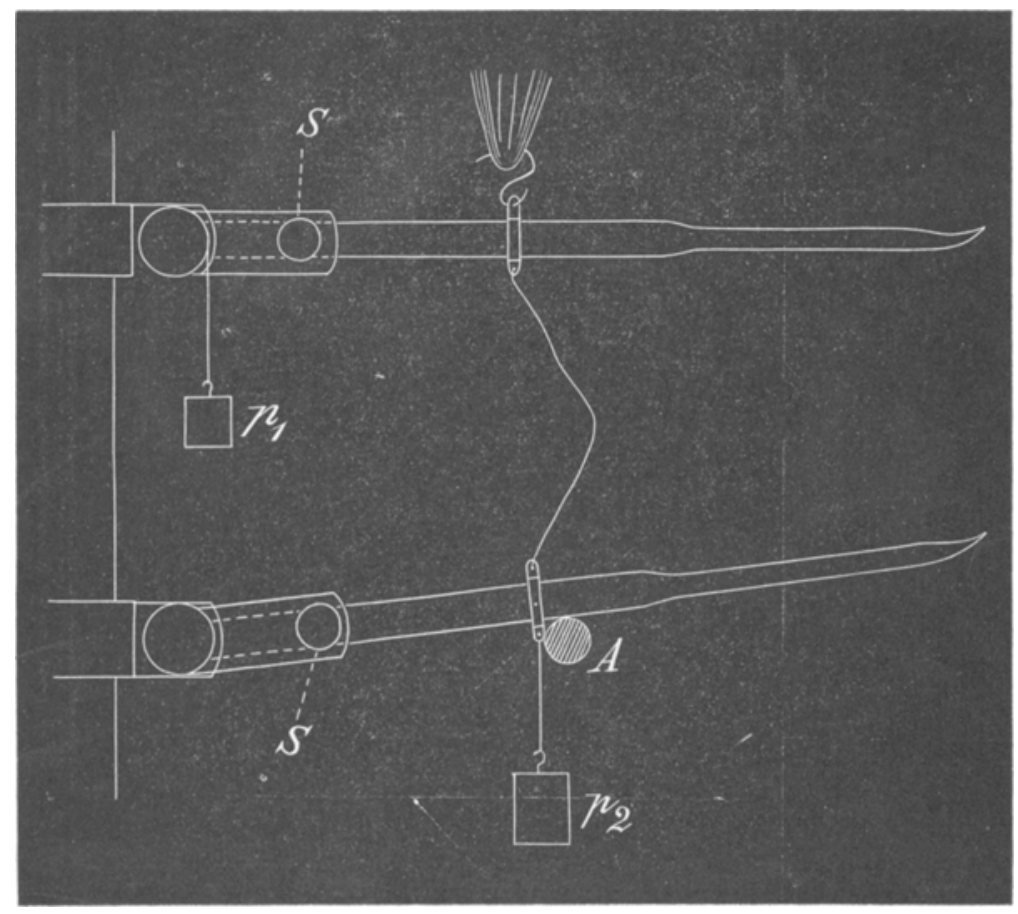

Stellt man den Unterstützungsstab höher ein, so dass der Faden zwischen den Hebeln ganz schlaff ist, so kann, wenn die Einstellung hoch genug war, der obere Hebel mit der Achsenbelastung gehoben werden, ohne dass der untere bewegt wird. In den Stellungen zwischen den beiden Extremen muss der Faden erst gespannt werden, d. h. der obere Hebel gehoben sein, ehe der untere mit der zweiten Last abgehoben wird. Variirt man die Stellung des

1) Dieser Hebel wurde nicht an der Achse belastet, weil die hierzu erforderlichen Gewichte zu gross gewesen wären. 
Stabes, so variirt man damit den Zeitpunkt, in dem das zweite Gewicht gehoben wird.

In analoger Weise könnte man den Muskel beliebig polytonische Zuckungen ausführen lassen, indem man noch eine dritte, vierte und weitere Belastung während der Zuckung wirken liesse. Man sieht leicht, dass die Versuchsanordnung noch weiterer Variationen fähig ist. Man kann die ditonische Zuckung einerseits leicht in eine Ueberlastungszuckung - durch tiefe Eiristellung des Unterstützungsstabes - überführen, man kann sie andererseits mit wachsendem Gewichte der zweiten Last in eine v. Kries' sche Anschlagszuckung erster Combination ${ }^{1}$ ) verwandeln. Diese kann als ein specieller Fall der ditonischen Zuckung angesehen werden, denn bei ihr wird durch Anschlag des Verkürzungsschreibers an einen festen Widerstand der Muskel an seiner weiteren Verkürzung verhindert.

Die Versuche wurden so ausgeführt, dass zuerst eine ganz monotonisch verlaufende Zuckung auf einer Trommel verzeichnet wurde. Dann wurde eine Zuckung gezeichnet, bei der das zweite Gewicht sehr früh nach Beginn der Contraction gehoben wurde. Bei den folgenden Zuckungen war die Einstellung immer höher, der "Umschlagspunkt", in welchem die niedere Spannung in die höhere übergeht, fiel immer später.

Bei den ditonischen Zuckungen zeiehnen die Hebel zwei Curven untereinander. Die Höhe der oberen ist die Höhe, auf welche die Anfangslast, die der unteren die Höhe, auf welche die zweite Last gehoben wurde. Aus beiden lässt sich unmittelbar die geleistete Arbeit berechnen. - Auf diese Weise sind die in den folgenden Tabellen zusammengestellten Resultate erhalten.

Die beiden Hebel bestehen aus zwei $22,5 \mathrm{~cm}$ langen und $1 \mathrm{~cm}$ hohen Aluminiumstreifen, die sich nach dem Zeichenende hin verjüngen und in einer feinen Spitze auslaufen, mit der gezeichnet wurde. Das genaue Anlegen der Hebel an die Trommel geschah durch Verstellen einer am axialen Ende jedes Hebels befindlichen, in einer Mutter beweglichen Schraube (in der Figur mit $\mathrm{S}$ bezeichnet). Der Spitze dieser liegt der Hebel federnd an und wird daher durch Vor- oder Zurückschrauben der Trommel genähert resp. entfernt. -

1) v. Kries, Untersuchungen zur Mechanik des quergestreiften Muskeis. Du Bois' Archiv 1892. 
Ueb. d. Einfluss d. Spannungszunahme während d. Zuckung a. d. Arbeitsl. etc. 597

Zur Befestigung der Verbindungsfäden zwischen den Hebeln dienten zwei mit Oesen versehene kleine Schieber aus Aluminium, welche den Hebeln fest ansitzen, so dass eine Verschiebung derselben wăhrend der Zuckung nicht stattfinden kann. -- Der Muskel griff $100 \mathrm{~mm}$ von der Achse entfernt am Hebel an; der Radius der Rolle am isotonischen Schreibhebel betrug $7 \mathrm{~mm}$. Die Belastung, die der Muskel durch einen Hebel erfuhr, entsprach einem Gewichte von $5,5 \mathrm{~g}$; die Belastung durch den Haken zwischen Muskel und oberem Hebel 0,6 g. Es addiren sich also zur Anfangsbelastung (mit $p_{1}$ bezeichnet) nach $6,1 \mathrm{~g}$, zur 2 . Belastung $\left(p_{2}\right)$ noch $5,5 \mathrm{~g}$. Die Höhen sind in $2^{1 / 4}$ facher Vergrösserung gezeichnet. Die Reizung geschah durch Inductionsöffnungsschlag eines D u Boi s'schen Schlitteninductoriums; zunächst wurde stets maximal gereizt. In den Tabellen, sowie in den später zu besprechenden Curven ist mit $m$ die monotonische Zuckung, mit $d_{1} d_{2} d_{3} \ldots$ die ditonische Zuckung bezeichnet, mit $h_{1}$ die Höhe, auf welche die Anfangslast gehoben wurde, mit $h_{2}$ die zur zweiten Last gehörige Höhe. $p_{1}$ ist die Anfangslast, $p_{2}$ die während der Zuckung zu hebende. In den beiden mit "Arbeit“ überschriebenen Rubriken stehen in gmm die Arbeitsleistungen, links aus den unter $p_{1}$ und $p_{2}$ angegebenen Belastungen berechnet, rechts die corrigirten Werthe mit Berücksichtigung der thatsächlichen Belastung $\left(p_{1}+6,1, p_{2}+5,5\right)$.

21. Januar 1897.

I.

\begin{tabular}{l|l|c|c|c|c|l}
\hline & $h_{1}$ & $h_{2}$ & $p_{1}$ & $p_{2}$ & \multicolumn{2}{|c}{ Arbeit in gmm } \\
\hline$m$ & 21 & - & 7 & - & 147 & 275,1 \\
$d_{1}$ & 12 & 10 & 7 & 50 & 584 & 712,2 \\
$d_{2}$ & 14 & 7 & 7 & 50 & 448 & 571,9 \\
$d_{3}$ & 13 & 1,5 & 7 & 50 & 166 & 253,55 \\
$d_{4}$ & 16 & - & 7 & - & 112 & 209,6 \\
$d_{5}$ & 20 & - & 7 & - & 140 & 262 \\
$m$ & 21 & - & 7 & - & 147 & 275,1 \\
$d_{2}$ & 17 & 5 & 7 & 50 & 369 & 500,2 \\
$d_{\mathbf{1}}$ & 15 & 11 & 7 & 50 & $\mathbf{6 5 5}$ & 807 \\
& & & & & &
\end{tabular}


22. Januar 1897.

II.

\begin{tabular}{l|l|r|r|r|r|r}
\hline & $h_{1}$ & $h_{2}$ & $p_{1}$ & $p_{2}$ & \multicolumn{2}{|c}{ Arbeit in gmm } \\
\hline$m$ & 23 & - & 14 & - & 322 & 462,3 \\
$d_{1}$ & 17 & 15 & 14 & 50 & 988 & 1174,2 \\
$d_{2}$ & 18 & 17 & 14 & 50 & 1102 & 1305,3 \\
$d_{3}$ & 19 & 6 & 14 & 50 & 566 & 714,9 \\
$d_{4}$ & 21 & - & 14 & - & 294 & 422,1 \\
$m$ & 22 & - & 14 & - & 308 & 442,2
\end{tabular}

22. Januar 1897.

III.

\begin{tabular}{c|c|c|c|c|c|c}
\hline & $h_{1}$ & $h_{2}$ & $p_{1}$ & $p_{2}$ & \multicolumn{2}{|c}{ Arbeit in gmm } \\
\hline$m$ & 22 & - & 7 & - & 154 & 288,2 \\
$d_{1}$ & 14 & 12 & 7 & 100 & 1298 & 1449,4 \\
$d_{2}$ & 16 & 9 & 7 & 100 & 1012 & 1159,1 \\
$d_{3}$ & 15 & 3 & 7 & 100 & 405 & 513 \\
$m$ & 20 & - & 7 & - & 140 & 262
\end{tabular}

22. Januar 1897

IV.

\begin{tabular}{c|c|c|c|c|c|c}
\hline & $h_{1}$ & $h_{2}$ & $p_{1}$ & $p_{2}$ & \multicolumn{2}{|c}{ Arbeit in gmm } \\
\hline$m$ & 21 & - & 14 & - & 294 & 422,1 \\
$d_{1}$ & 13 & 11 & 14 & 100 & 1282 & 1421,8 \\
$d_{2}$ & 15 & 7 & 14 & 100 & 910 & 1040 \\
$d_{3}$ & 14 & 3 & 14 & 100 & 496 & 597,9 \\
$m$ & 17 & - & 14 & - & 238 & 341,7
\end{tabular}

22. Januar 1897.

V.

\begin{tabular}{l|l|l|l|l|l|l}
\hline & $h_{1}$ & $h_{2}$ & $p_{1}$ & $p_{2}$ & \multicolumn{2}{|c}{ Arbeit in gmm } \\
\hline$m$ & 19 & - & 14 & - & 266 & 381,9 \\
$d_{1}$ & 14 & 10 & 14 & 50 & 696 & 836,4 \\
$d_{2}$ & 14 & 0,5 & 14 & 50 & 221 & 309,15 \\
$d_{3}$ & 17 & - & 14 & - & 238 & 341,7 \\
$m$ & 19 & - & 14 & - & 266 & 381,9
\end{tabular}


Ueb. d. Einfluss d. Spannungszunahme während d. Zuckung a. d. Arbeitsl. etc. 599 23. Januar 1897. VI.

\begin{tabular}{l|l|l|l|l|l|l}
\hline & $h_{1}$ & $h_{2}$ & $p_{1}$ & $p_{2}$ & \multicolumn{2}{|c|}{ Arbeit in gmm } \\
\hline$m$ & 17 & - & 7 & - & 119 & 222,7 \\
$d_{1}$ & 12 & 7,5 & 7 & 100 & 834 & 948,45 \\
$d_{2}$ & 12 & 5 & 7 & 100 & 584 & 684,7 \\
$d_{\mathbf{3}}$ & 11,5 & 0,5 & 7 & 100 & 130,5 & 203,4 \\
$d_{2}$ & 12,5 & 5 & 7 & 100 & 587,5 & 691,25 \\
$d_{1}$ & 12,5 & 6,5 & 7 & 100 & 737,5 & 849,5
\end{tabular}

25. Januar 1897.

VII.

\begin{tabular}{l|c|c|c|c|c|c}
\hline & $h_{1}$ & $h_{2}$ & $p_{1}$ & $p_{2}$ & \multicolumn{2}{|c}{ Arbeit in gmm } \\
\hline$m$ & 19 & - & 7 & - & 133 & 248,9 \\
$d_{1}$ & 13 & 11,5 & 7 & 50 & 666 & 808,55 \\
$d_{2}$ & 14 & 10 & 7 & 50 & 598 & 738,4 \\
$d_{3}$ & 15 & 8 & 7 & 50 & 505 & 640,5 \\
$d_{4}$ & 15 & 5,5 & 7 & 50 & 380 & 501,75 \\
$d_{5}$ & 13 & 0,5 & 7 & 50 & 116 & 198,05 \\
$m$ & 15 & - & 7 & - & 105 & 196,5 \\
$d_{5}$ & 13 & 0,5 & 7 & 50 & 116 & 198,05 \\
$d_{4}$ & 14 & 5 & 7 & 50 & 348 & 460,9 \\
$d_{3}$ & 15 & 10 & 7 & 50 & 605 & 751,5 \\
$d_{2}$ & 14 & 12 & 7 & 50 & 698 & 849,4
\end{tabular}

25. Januar 1897.

VIII.

\begin{tabular}{l|l|l|l|l|l|l}
\hline & $h_{1}$ & $h_{2}$ & $p_{1}$ & $p_{2}$ & \multicolumn{2}{|c}{ Arbeit in gmm } \\
\hline$m$ & 17 & - & 14 & - & 238 & 341,7 \\
$d_{1}$ & 12 & 7 & 14 & 100 & 868 & 979,7 \\
$d_{2}$ & 12 & 6 & 14 & 100 & 768 & 874,2 \\
$d_{3}$ & 11 & 2 & 14 & 100 & 354 & 432,1 \\
$m$ & 12 & - & 14 & - & 168 & 241,2 \\
$d_{3}$ & 11 & 0,5 & 14 & 100 & 204 & 273,85 \\
$d_{2}$ & 11 & 3,5 & 14 & 100 & 504 & 590,35 \\
$d_{1}$ & 12 & 6 & 14 & 100 & 768 & 874,2
\end{tabular}

IX.

\begin{tabular}{c|c|c|c|c|c|c}
\hline & $h_{1}$ & $h_{2}$ & $p_{1}$ & $p_{2}$ & \multicolumn{2}{|c|}{ Arbeit in gmm } \\
\hline$m$ & 20 & - & 7 & - & 140 & 262 \\
$d_{1}$ & 18 & 8,5 & 7 & 150 & 1401 & 1557,55 \\
$d_{2}$ & 12 & 4 & 7 & 150 & 684 & 779,2 \\
$d_{3}$ & 11,5 & 1 & 7 & 150 & 230,5 & 306,15 \\
$d_{4}$ & 14 & - & 7 & - & 98 & 183,4 \\
$m$ & 21 & - & 7 & - & 147 & 275,1
\end{tabular}


$\mathrm{X}$.

\begin{tabular}{l|l|l|l|l|l|r}
\hline & $h_{1}$ & $h_{2}$ & $p_{1}$ & $p_{2}$ & \multicolumn{2}{|c}{ Arbeit in gmm } \\
\hline$m$ & 20,5 & - & 7 & - & 143,5 & 268,55 \\
$d_{1}$ & 12,5 & 7 & 7 & 130 & 997,5 & 1112,25 \\
$d_{2}$ & 11,5 & 2,5 & 7 & 130 & 405,5 & 489,40 \\
$d_{3}$ & 11 & 1,5 & 7 & 130 & 272 & 347,35 \\
$d_{4}$ & 12,5 & 0,5 & 7 & 130 & 152,5 & 281,50 \\
$d_{5}$ & 15 & - & 7 & - & 105 & 196,5 \\
$m$ & 20 & - & 7 & - & 140 & 262
\end{tabular}

$\mathrm{XI}$.

\begin{tabular}{c|c|c|c|c|c|c}
\hline & $h_{\mathbf{1}}$ & $h_{\mathbf{2}}$ & $p_{\mathbf{1}}$ & $p_{\mathbf{2}}$ & \multicolumn{2}{|c}{ Arbeit in gmm } \\
\hline$m$ & 22 & - & 7 & - & 154 & 288,2 \\
$d_{\mathbf{1}}$ & 10 & 6 & 7 & 200 & 1270 & 1364 \\
$d_{\mathbf{2}}$ & 10 & 3,5 & 7 & 200 & 770 & 850,25 \\
$d_{\mathbf{3}}$ & 9,5 & 1,5 & 7 & 200 & 366,5 & 432,70 \\
$d_{4}$ & 11,5 & 0,5 & 7 & 200 & 180,5 & 253,40 \\
$d_{5}$ & 16,5 & - & 7 & - & 115,5 & 216,15 \\
$m$ & 21,5 & - & 7 & - & 150,5 & 281,65
\end{tabular}

XII.

\begin{tabular}{l|c|c|c|c|c|c}
\hline & $h_{1}$ & $h_{2}$ & $p_{1}$ & $p_{2}$ & \multicolumn{2}{|c}{ Arbeit in gmm } \\
\hline$m$ & 22 & - & 7 & $:-$ & 154 & 288,2 \\
$d_{1}$ & 8 & 4 & 7 & 230 & 976 & 1046,8 \\
$d_{2}$ & 8 & 2 & 7 & 230 & 516 & 575,8 \\
$d_{3}$ & 9 & 1 & 7 & 230 & 293 & 353,4 \\
$d_{4}$ & 11,5 & 0,5 & 7 & 230 & 195,5 & 268,4 \\
$d_{5}$ & 17 & - & 7 & - & 119 & 222,7 \\
$m$ & 21 & - & 7 & - & 147 & 275,1
\end{tabular}

Aus dem Vergleich der Zahlen geht unmittelbar hervor, dafs bei ditonischer Zuckung mehr Arbeit geleistet werden kann als bei monotonischer, und zwar wird eine um so grössere Arbeit geleistet, je früher die zweite Last gehoben wird (bei $d_{1}$ oder $d_{2}$ Arbeitsmaximum); nähert sich die Hebung der zweiten Last dem Maximnm der Zuckung, so sinkt die Arbeit unter die der monotonischen Zuckung; demgemäss muss bei Ueberlastungszuckung die Arbeitsleistung unter sonst gleichen Verhältnissen noch grösser 
Ueb. d. Einfluss d. Spannungszunahme während d. Zuckung a. d. Arbeitsl. etc. 601 sein als bei ditonischer mit Mehrbelastung kurz nach begonnener Zusammenziehung. Dies ist auch thatsächlich der Fall, wie die folgenden Tabellen zeigen. Mit $\ddot{u}$ ist die Ueberlastungszuckung bezeichnet.

I.

\begin{tabular}{c|c|c|c|c|c|c}
\hline & $h_{1}$ & $h_{2}$ & $p_{1}$ & $p_{2}$ & \multicolumn{2}{|c|}{ Arbeit in gmm } \\
\hline$\ddot{u}$ & 12 & 10 & 7 & 100 & 1084 & 1212,2 \\
$d_{1}$ & 14 & 9 & 7 & 100 & 998 & 1132,9 \\
$d_{2}$ & 14 & 7 & 7 & 100 & 798 & 921,9 \\
$d_{3}$ & 14 & 6,5 & 7 & 100 & 748 & 869,15
\end{tabular}

II.

\begin{tabular}{|c|c|c|c|c|c|c|}
\hline & $h_{1}$ & $h_{2}$ & $p_{1}$ & $p_{2}$ & \multicolumn{2}{|c|}{ Arbeit in $\mathrm{gmm}$} \\
\hline$\ddot{u}$ & 9 & 6,5 & 7 & 200 & 1363 & 1453,65 \\
\hline$d_{1}$ & 10 & 5 & 7 & 200 & 1070 & 1158,5 \\
\hline$d_{2}$ & 10 & 2 & 7 & 200 & 470 & 542 \\
\hline
\end{tabular}

III.

\begin{tabular}{r|r|r|r|r|r|r}
\hline & $h_{1}$ & $h_{2}$ & $p_{1}$ & $p_{2}$ & \multicolumn{2}{|c}{ Arbeit in gmm } \\
\hline$\ddot{u}$ & 11 & 10 & 14 & 100 & 1154 & 1276,1 \\
$d_{1}$ & 10 & 9 & 14 & 100 & 1040 & 1160,5 \\
$d_{2}$ & 13 & 7 & 14 & 100 & 882 & 999,8 \\
$d_{3}$ & 13 & 5 & 14 & 100 & 682 & 788,8
\end{tabular}

IV.

\begin{tabular}{l|l|l|l|l|l|l}
\hline & $h_{1}$ & $h_{2}$ & $p_{1}$ & $p_{2}$ & \multicolumn{2}{c}{ Arbeit in gmm } \\
\hline$\ddot{u}$ & 13 & 11,5 & 7 & 50 & 666 & 808,55 \\
$d_{1}$ & $\mathbf{1 3}$ & 10 & 7 & 50 & 591 & 725,3 \\
$d_{2}$ & 13,5 & 9,5 & 7 & 50 & 569,5 & 704,1 \\
$d_{3}$ & 13,5 & 7 & 7 & 50 & 444,5 & 565,35
\end{tabular}

V.

\begin{tabular}{l|l|l|l|l|l|l}
\hline & $h_{1}$ & $h_{2}$ & $p_{1}$ & $p_{2}$ & \multicolumn{2}{|c}{ Arbeit in gmm } \\
\hline$\ddot{u}$ & 12 & 11 & 14 & 50 & 718 & 851,7 \\
$d_{1}$ & 12,5 & 9 & 14 & 50 & 625 & 750,75 \\
$d_{2}$ & 13 & 8 & 14 & 50 & 582 & 705,3
\end{tabular}


In den obigen Versuchen wuchs mit der Grösse der zweiten Belastung die Arbeitsleistung. Natürlich muss dies seine Grenzen haben und es mufs bei einer bestimmten Belastung ein Arbeitsmaximum geben. Um diesen Grenzfall zu erreichen, wurden bei gleichbleibender Einstellung wachsende Gewichte an den unteren Hebel angehängt und die Arbeitsleistung bestimmt. Um den Einfluss der Ermüdung aufzuheben, wurden zwei Versuchsreihen angestellt - , indem der Muskel einmal mit wachsender und "einmal mit abnehmender zweiter Belastung arbeitete. Die folgenden Zahlen sind Mittelzahlen aus zwei solchen Versuchen.

$$
\begin{aligned}
& \text { Arbeit in gmm } \\
& \text { Monotonie: } p_{1}=7 \text {. . . . . . . } 140 \quad 262 \\
& \text { Ditonie: } \quad p_{2}=10 \text {. . . . . . . } 258,5 \quad 425,45 \\
& 20 \text {. . . . . } 372,5 \quad 533,65 \\
& 30 \text {. . . . . . } 454,75 \quad 607,35 \\
& 40 \text {. . . . . . } 530,25 \quad 671,25 \\
& 50 \text {. . . . . . } 594,25 \quad 729,45 \\
& 60 \text {. . . . . . } 641,5 \quad 770,9 \\
& 70 \text {. . . . . . . } 725,5 \quad 843,3 \\
& 80 \text {. . . . . . . } 692,75 \quad 794,4 \\
& 90 \text {. . . . } 696,75 \quad 798,95 \\
& 100 \text {. . . . . . } 635,75 \quad 727,60 \\
& 110 \text {. . . . . . . } 630,5 \quad 708,0 \\
& 120 \text {. . . . . . . } 585,25 \quad 656,95 \\
& 130 \text {. . . . . . } 525 \quad 590,9 \\
& 150 \text {. . . . . . . } 400,5 \quad 454,8 \\
& 160 \text {. . . . . . . } 379,5 \quad 433,8 \\
& 200 \text {. . . . . 204,25 258,55 }
\end{aligned}
$$

Bei 230 Anschlagszuckung.

Die Zahlen zeigen, dass mit zunehmender zweiter Belastung die Arbeitsleistungen erst rasch, dann langsamer wachsen, ein Maximum erreichen und dann langsam wieder abnehmen.

Was die Gesammthöhe der ditonischen Zuckungen betrifft $\left(h_{1}\right)$, so nimmt sie mit der Höhe der Einstellung zu, d. h. sie wird grösser, in einem je späteren Momente der Zuckung die Spannungszunahme stattfindet. Dies stimmt mit einer Beobachtung von v. Kries') ferner mit der Sogalla's ${ }^{2}$ ) über die Hubhöhen der Schleuder-

1) v: Kries, Untersuchungen zur Mechanik des quergestreiften Muskels. Du Bois Archiv 1880 S. 368.

2) Sogalla, Beiträge zur mechanischen Analyse der Muskelzuckungscurve. Würzburg 1889. 
zuckungen überein. In der Arbeit Sogalla's findet sich auch eine Angabe über die Arbeitsleistungèn des Muskels bei Schleuderzuckungen. Indem Sogalla einen Schleuderhebel entweder gleich bei Beginn der Contraction, oder erst im Verlaufe derselben von dem mit dem Muskel verbundenen Schreibhebel, wegschleudern liess, konnte er aus der lebendigen Kraft des Schleuderhebels einen Anhaltspunkt für die vom Muskel im Momente der Schleuderung geleistete Arbeit gewinnen. Die Trägheit des Schleuderhebels konnte noch durch Gewichte erhöht werden. Indem er so den Hebel mit oder ohne Massen in verschiedenen Momenten der Contraction schleudern liess, fand er, dass „die lebendige Kraft zunächst mit wachsender Trägheit steigt, gleichviel, ob die Schleuderung bereits bei der natürlichen Länge des rubenden Muskels beginnt, oder erst dann, wenn der Muskel einen Theil seiner Verkürzung isotonisch durehlaufen hat". Es steigt also auch unter diesen - von unserer Anordnung verschiedenen Umständen - die Arbeitsleistung des Muskels zu einem Maximum an und würde jedenfalls nach Erreichung des Grenzfalles mit zunehmender Trägheit wieder absinken.

Bisher wurde der Muskel stets maximal gereizt; es interessirt nun zu wissen, ob das Verhältniss dasselbe bleibt, wenn man untermaximale und schliesslich minimale Reize, $d . h$. solche, die eben noch eine Hebung beider Gewichte hervorbringen, in den Muskel hineinschickt. Es könnte dies für die Vorstellung, die man sich von der Wirkung der Spannungszunahme während der Zuckung macht, von Bedeutung sein. A priori sind zwei Möglichkeiten denkbar. Entweder, das Verhältniss der Arbeitsleistungen bei ditonischer und monotoniseher Zuckung bleibt auch bei minimalen Reizen so wie bei maximalen, dann könnte man annehmen, dass Reizwirkung und die Wirkung der Spannungszunahme während der Zuckung sich einfach summiren; oder es wird, wenn die Reize minimal sind, bei ditonischer Zuckung nicht mehr, vielleicht sogar weniger Arbeit geleistet als bei monotonischer, dann muss das Verhältniss von Reizwirkung und Spannungsvermehrung ein complicirteres sein.

Die Versuche zeigen, dass bei untermaximalen Reizen das Verhältniss zunächst dasselbe bleibt, wie bei maximalen. Ein Blick auf die folgenden Tabellen lehrt, dass auch hier bei ditonischer Zuckung mehr Arbeit geleistet werden kann als bei monotonischer. Nur in einem Falle kehrte sich das Verhältniss um (siehe Tab. IX). In diesem 
Versuche, ebenso wie in Versuch $\mathrm{X}$, wo einmal ein Vergleich der corrigirten Werthe (IX $m=129,03$ und $d_{1}=109,35$ ) und anderseits im Versuch $\mathrm{X}$ die nicht corrigirten $\left(m=45,5\right.$ und $\left.d_{1}=33,0\right)$ eine Umkehrung des Verhältnisses ergiebt, muss die Reizwirkung eine sehr geringe gewesen sein. Die beiden Versuche stehen also auf der Grenze zwischen den Versuchen mit untermaximaler und minimaler Reizung, welch' letztere im Folgenden besprochen werden sollen.

\begin{tabular}{l|l|l|l|l|l|l}
\multicolumn{2}{c}{ Rolienentfernung 210. } & \multicolumn{1}{l}{} \\
\hline & $h_{1}$ & $h_{2}$ & $p_{1}$ & $p_{2}$ & \multicolumn{2}{c}{ Arbeit in gmm } \\
\hline$m$ & 17 & - & 3,5 & - & 59,5 & 163,2 \\
$d_{1}$ & 10,5 & 6,5 & 3,5 & 30 & 231,75 & 331,55 \\
$d_{2}$ & 10 & 5 & 3,5 & 30 & 185 & 273,5 \\
$d_{3}$ & 8,5 & 1,5 & 3,5 & 30 & 74,75 & 134,85 \\
$d_{4}$ & 9 & 0,5 & 3,5 & 30 & 46,5 & 104,15 \\
$d_{5}$ & 10,5 & - & 3,5 & - & 36,75 & 100,8 \\
$m$ & 15,5 & - & 3,5 & -- & 54,25 & 148,8
\end{tabular}

Rollenentfernung 190.

II.

\begin{tabular}{l|l|l|l|l|l|l}
\hline & $h_{1}$ & $h_{2}$ & $p_{1}$ & $p_{2}$ & \multicolumn{2}{|c}{ Arbeit in gmm } \\
\hline$m$ & 18 & - & 3,5 & - & 68 & 172,8 \\
$d_{1}$ & 13 & 8 & 3,5 & 20 & 205,5 & 328,8 \\
$d_{2}$ & 13 & 7 & 3,5 & 20 & 185,5 & 303,3 \\
$d_{3}$ & 13,5 & 5 & 3,5 & 20 & 147,25 & 257,1 \\
$d_{4}$ & 13 & 4 & 3,5 & 20 & 125,5 & 226,8 \\
$d_{5}$ & 10,5 & 0,5 & 3,5 & 20 & 46,75 & 113,55 \\
$d_{6}$ & 13 & - & 3,5 & - & 45,5 & 124,8 \\
$m$ & 16,5 & - & 3,5 & - & 57,75 & 158,4
\end{tabular}

Rollenentfernung 194.

III.

\begin{tabular}{l|r|c|c|c|c|c}
\hline \hline & $h_{1}$ & $h_{2}$ & $p_{1}$ & $p_{2}$ & \multicolumn{2}{|c}{ Arbeit in gmm } \\
\hline$m$ & 16 & - & 3,5 & - & 56 & 153,6 \\
$d_{1}$ & 9 & 4 & 3,5 & 40 & 191,5 & 268,4 \\
$d_{2}$ & 9 & 3 & 3,5 & 40 & 151,5 & 222,9 \\
$d_{3}$ & 7 & 1,5 & 3,5 & 40 & 84,5 & 135,45 \\
$d_{4}$ & 10 & - & 3,5 & - & 35 & 96 \\
$m$ & 14 & - & 3,5 & - & 49 & 134,4
\end{tabular}


Ueb. d. Einfluss d. Spannungszunahme während d. Zuckung a. d. Arbeitsl. etc. 605 Rollenentfernung 274 .

IV.

\begin{tabular}{l|l|l|l|l|l|l}
\hline \hline & $h_{1}$ & $h_{2}$ & $p_{1}$ & $p_{2}$ & \multicolumn{2}{|c}{ Arbeit in gmm } \\
\hline$m$ & 5 & - & 3,5 & - & 17,5 & 48 \\
$d_{1}$ & 2,5 & 2 & 3,5 & 23 & 54,75 & 81
\end{tabular}

\begin{tabular}{c|c|c|c|c|c|c}
\multicolumn{3}{c}{ Rollenentfernung 264. } & \multicolumn{2}{c}{ V. } \\
\hline & $h_{1}$ & $h_{2}$ & $p_{1}$ & $p_{2}$ & \multicolumn{2}{|c}{ Arbeit in gmm } \\
\hline$m$ & 11,5 & - & 3,5 & - & $\begin{array}{r}40,25 \\
111,25\end{array}$ & 110,4 \\
$d_{1}$ & 5,5 & 4 & 3,5 & 28 & 166,8
\end{tabular}

\begin{tabular}{|c|c|c|c|c|c|}
\hline$h_{1}$ & $h_{2}$ & $p_{1}$ & $p_{2}$ & \multicolumn{2}{|c|}{ Arbeit in $\mathrm{gmm}$} \\
\hline $\begin{array}{r}10,5 \\
5,5 .\end{array}$ & $\overline{4}$ & $\begin{array}{l}3,5 \\
3,5\end{array}$ & $\overline{23}$ & $\begin{array}{r}36,75 \\
111,25\end{array}$ & $\begin{array}{l}100 \\
166\end{array}$ \\
\hline
\end{tabular}

Rollenentfernung 235. VII.

\begin{tabular}{l|r|l|l|l|l|l}
\hline & $h_{1}$ & $h_{2}$ & $p_{1}$ & $p_{2}$ & \multicolumn{2}{|c}{ Arbeit in gmm } \\
\hline$m$ & 13 & - & 3,5 & - & $\begin{array}{l}45,5 \\
96,5\end{array}$ & $\begin{array}{l}124,8 \\
134,65\end{array}$
\end{tabular}

Rollenentfernung 227. VIII.

\begin{tabular}{l|c|c|c|c|c|c}
\hline & $h_{1}$ & $h_{2}$ & $p_{1}$ & $p_{2}$ & \multicolumn{2}{|c}{ Arbeit in gmm } \\
\hline$m$ & 13,5 & - & 3,5 & - & $\begin{array}{c}47,25 \\
130\end{array}$ & $\begin{array}{c}129,6 \\
174,25\end{array}$ \\
\hline$d_{1}$ & 5 & 2,5 & 3,5 & 45 & 130
\end{tabular}

Rollenentfernung 230.

IX.

\begin{tabular}{|c|c|c|c|c|c|c|}
\hline & $h_{1}$ & $h_{2}$ & $p_{1}$ & $p_{2}$ & \multicolumn{2}{|c|}{ Arbeit in $\mathrm{gmm}$} \\
\hline $\begin{array}{l}m \\
d_{1}\end{array}$ & $\begin{array}{r}13,5 \\
3,5\end{array}$ & $\overline{1, \dot{5}}$ & $\begin{array}{l}3,5 \\
3,5\end{array}$ & $\overline{45}$ & $\begin{array}{l}47,25 \\
79,75\end{array}$ & $\begin{array}{l}129,6 \\
109,35\end{array}$ \\
\hline
\end{tabular}


Rollenentfernung 235.

\begin{tabular}{|c|c|c|c|c|c|c|}
\hline & $h_{1}$ & $h_{2}$ & $p_{1}$ & $p_{z}$ & \multicolumn{2}{|c|}{ Arbeit in $\mathrm{gmm}$} \\
\hline $\begin{array}{l}m \\
d_{1}\end{array}$ & $\begin{array}{r}13 \\
3\end{array}$ & $\overline{0,5}$ & $\begin{array}{l}3,5 \\
3,5\end{array}$ & $\overline{45}$ & $\begin{array}{l}45,5 \\
33\end{array}$ & $\begin{array}{c}124,8 \\
54,05\end{array}$ \\
\hline
\end{tabular}

Minimale Reiznng.

Als minimale Reize werden diejenigen bezeichnet, die eine nur geringe Erhebung des zweiten Gewichtes zu bewirken im Stande sind. Die Höhe $h_{2}$ liegt dann zwischen 0,5 und $1,5 \mathrm{~mm}$.

Rollenentfernung 206.

I.

\begin{tabular}{c|c|c|c|c|c|r}
\hline \hline & $h_{1}$ & $h_{2}$ & $p_{1}$ & $p_{2}$ & \multicolumn{2}{|c}{ Arbeit in gmm } \\
\hline$m$ & 14 & - & 3,5 & - & 49,0 & 134,4 \\
$d_{1}$ & 3,0 & 1,0 & 3,5 & 20 & 30,5 & 54,3
\end{tabular}

Rollenentfernung 203.

II.

\begin{tabular}{c|c|c|c|c|c|c}
\hline & $h_{1}$ & $h_{2}$ & $p_{1}$ & $p_{2}$ & \multicolumn{2}{|c}{ Arbeit in gmm } \\
\hline$m$ & 13 & - & 3,5 & - & $\begin{array}{c}45,5 \\
20,5\end{array}$ & $\begin{array}{c}124,8 \\
41,55\end{array}$ \\
\hline
\end{tabular}

Rollenentfernung 207,5 .

III.

\begin{tabular}{|c|c|c|c|c|c|c|}
\hline & $h_{1}$ & $h_{2}$ & $p_{\mathbf{1}}$ & $p_{2}$ & \multicolumn{2}{|c|}{ Arbeit in $\mathbf{g m m}$} \\
\hline $\begin{array}{l}m \\
d_{1}\end{array}$ & $\begin{array}{l}8,5 \\
3,0\end{array}$ & $\overline{1,5}$ & $\begin{array}{l}3,5 \\
3,5\end{array}$ & $\overline{10}$ & $\begin{array}{l}29,75 \\
25,5\end{array}$ & $\begin{array}{l}81,6 \\
52,05\end{array}$ \\
\hline
\end{tabular}

Rollenentfernung 206,5 .

IV.

\begin{tabular}{|c|c|c|c|c|c|c|}
\hline & $h_{1}$ & $h_{2}$ & $p_{1}$ & $p_{2}$ & \multicolumn{2}{|c|}{ Arbeit in $\mathrm{gmm}$} \\
\hline$m$ & 12 & - & 3,5 & - & 42,0 & 115,2 \\
\hline$d_{1}$ & 3,0 & 1,0 & 3,5 & 10 & 20,5 & 44, \\
\hline
\end{tabular}


Ueb. d. Einfluss d. Spannungszunahme während d. Zuckung a. d. Arbeitsl. etc. 607 Rollenentfernung 220 .

V.

\begin{tabular}{|c|c|c|c|c|c|c|}
\hline & $h_{1}$ & $h_{2}$ & $p_{1}$ & $p_{2}$ & \multicolumn{2}{|c|}{ Arbeit in gmm } \\
\hline $\begin{array}{l}m \\
d_{1}\end{array}$ & $\begin{array}{r}10,5 \\
3,0\end{array}$ & $\overline{2,5}$ & $\begin{array}{l}3,5 \\
3,5\end{array}$ & $\overline{20}$ & $\begin{array}{l}36,75 \\
60,75\end{array}$ & $\begin{array}{r}100,8 \\
92,55\end{array}$ \\
\hline
\end{tabular}

Rollenentfernung 216,5 .

VI.

\begin{tabular}{l|l|l|l|l|l|l}
\hline & $h_{\mathfrak{1}}$ & $h_{2}$ & $p_{1}$ & $p_{2}$ & \multicolumn{2}{|c}{ Arbeit in gmm } \\
\hline$m$ & 8,5 & - & 3,5 & - & $\begin{array}{l}29,75 \\
32,25\end{array}$ & $\begin{array}{l}81,6 \\
59,1\end{array}$
\end{tabular}

Rollenentfernung 184,5 .

VII.

\begin{tabular}{c|c|c|c|c|c|c}
\hline \hline & $h_{\mathbf{1}}$ & $h_{\mathbf{2}}$ & $p_{\mathbf{1}}$ & $p_{2}$ & \multicolumn{2}{|c}{ Arbeit in gmm } \\
\hline$m$ & 18 & - & 3,5 & - & 63,0 & 172,8 \\
$d_{\mathbf{1}}$ & 3,5 & 0,5 & 3,5 & 20 & 22,25 & 46,35
\end{tabular}

Rollenentfernung 184,5.

VIII.

\begin{tabular}{c|c|c|c|c|c|c}
\hline \hline & $h_{1}$ & $h_{2}$ & $p_{1}$ & $p_{2}$ & \multicolumn{2}{|c}{ Arbeit in gmm } \\
\hline$m$ & 13 & - & 3,5 & -- & 45,5 & 124,8 \\
$d_{1}$ & 3,0 & 1,0 & 3,5 & 20 & 30,5 & 54,3
\end{tabular}

Die Tabellen zeigen, dass bei minimaler Reizung bei der ditonischen Zuckung weniger Arbeit geleistet wurde, als bei der entsprechenden monotonischen. Im. Versuch V und VI zeigen die nicht corrigirten und corrigirten Werthe für die Arbeitsleistung eine auffallende Differenz, indem bei den nicht corrigirten sich das Verhältnis zwischen den bei $m$ und $d_{1}$ geleisteten Arbeiten umkehrt. Es deutet das wohl darauf hin, dass in diesen Fällen die Reizung eben gerade noch minimal war. Es gelingt bei der wechselnden Erregbarkeit des Muskels nicht immer leicht, den Minimalreiz zu finden. Immerhin folgt aus den Versuchen mit minimaler und maximaler Reizung, dass die Spannungsvermehrung während der Zuckung nur dann eine vermehrte Arbeitsleistung bewirkt, wenn die Reize nicht unter ein gewisses 
Minimum herabsinken. Wie man sich hiernach die Wirkung von Reiz und Spannungsvermehrung während der Zuckung auf die der Contraction zu Grunde liegenden Processe vorzustellen hat, muss vorläufig noch dahingestellt bleiben, jedenfalls summiren sich die beiden Wirkungen nicht in einfacher Weise. Es müsste zunächst durch thermische Versuche festgestellt werden, ob bei ditonischer Zuckung grössere Energiemengen verbraucht werden als bei isotonischer.

\section{Gestalt der ditomischen Zuckungscurven.}

Es war nun weiter von Interesse, wie die zweite Spannung die Gestalt der Zuckungseurve verändern würde, und zu untersuchen, ob der "Umschlagspunkt" sich deutlich markirt, ob die Lage des Maximum sich verschiebe und ob schliesslich das ganze Areal der Zuckungscurven verkürzt oder verlängert werde. - $\mathrm{Zu}$ diesem Zwecke wurden die Zuckungen auf einer rotirenden Trommel verzeichnet.

Bei langsamer Trommelrotation $\left(u=10^{\prime \prime}\right)$ gezeichnete Curven (siehe Tafel VI Curvenfolge 1 und Curvenfolge $3 a$ ) zeigen im aufsteigenden Schenkel an einer bei $d_{1}$ weit unten, bei $d_{2}, d_{3} \ldots \ldots$ weiter oben gelegenen Stelle einen Knick. Dieser entspricht dem Momente, in welchem die erste Spannung in die höheren Grades übergeht. Fällt dieser bei hoher Einstellung der zweiten Last erst in den Zeitpunkt, wo der Muskel sich fast ad maximum verkürzt hat, so sieht man eine Verbreiterung des Curvengipfels; der Fall der Ansehlagszuckung mit hoher Einstellung des Anschlages ist erreicht. (S. Taf. VI Curvenfolge $1 d_{4}$ und $d_{5}$ und Curvenfolge $3 a^{a} d_{7}$ ). - Bei rascherer Trommelrotation gezeichnete Curven $\left(u=1^{\prime \prime}\right)$ zeigen das Verhalten an der Stelle des Umschlages deutlicher (s. Taf. VII Curvenfolge 2 a und 2 b). Auf eine, je nach der Einstellung grössere oder kleinere Strecke steilen Anstiegs folgt eine kurze Strecke, in welcher sich der Muskel nicht weiter verkürzt. Die Curve läuft der Abscisse nahezu parallel. (Die geringe Abweichung nach unten kann natürlich nicht etwa auf einer Dehnung beruhen, sondern muss als ein Fehler angesehen werden, bedingt durch die Elasticität des Schreibhebels und des Fadens.) Dann sieht man einen zweiten Anstieg. Es folgt also auf die isotonische Con- 
Ueb. d. Einfluss d. Spannungszunahme während d. Zuckung a. d. Arbeitsl. etc. 609

traktion ein isometrisches Schaltstück, dann eine weitere Contraktion unter vermehrter Spannung.

Der Punkt des zweiten Anstiegs fällt natürlich zeitlich mit dem Momente zusammen, in welchem sich die vom unteren Hebel gezeichnete Curve von ihrer Abscisse abhebt. Die Lage der beiden Punkte der oberen und unteren Curve lässt sich direkt mit einander vergleichen, wenn man die vor jeder Curve befindlichen Marken, welche die Stellung der beiden Hebel zu einander angeben, berücksichtigt.

Im absteigenden Curvenschenkel ist der „Umschlagspunkt" nicht deutlich zu sehen. Der Spannungswechsel markirt sich hier, wie aus den im Folgenden zu besprechenden Spannungscurven zu entnehmen ist, nicht scharf.

\section{Spannungseurven.}

Um den Spannungsablauf direet zur Anschauung zu bringen, wurde mit der oben angegebenen Vorrichtung noch der FickSchönlein'sche Spannungszeichner verbunden. Derselbe wurde so angebracht, dass der Muskel mit seinem oberen Ende mit dem Spannungszeichner, mit seinem unteren mit dem Längenzeichner verbunden war. So erhält man zwei Curven unter einander: oben die Spannungs- und unten die Verkürzungscurven. (Der unterste Hebel konnte wegen Platzmangel an der Trommel nicht mitschreiben.) An den bei langsamer Trommelrotation $\left(u=10^{\prime \prime}\right)$ gezeichneten Curven (S. Taf. VI Curvenfolge 3 a) sieht man den "Umschlagspunkt" im aufsteigenden Curvenschenkel scharf, im absteigenden entweder gar nicht, oder nur ganz undeutlich. Es wird dies wohl darauf beruhen, dafs die Erschlaffung viel allmäliger stattfindet als die Verkürzung und so der Uebergang der Spannung böheren in den niederen Grades in der Curve nicht so deutlich wiedergegeben wird.

Im Falle der Anschlagszuckung mit hoher Einstellung (S. Taf. VI Curvenfolge $3 \mathrm{a} \quad d_{\tau}$ ) sieht man die kurz dauernde Spannungszunahme auf dem Gipfel der Contraktion. Eine bei raseher Trommelrotation gezeichnete Curve $3 \mathrm{~b}$ zeigt dies Verhalten noch deutlicher. Auch hier lassen sich die einzelnen Punkte der beiden Curven mit Hülfe der rorgezeichneten Marken, welche die Hebelstellung angeben, mit einander leicht vergleichen. 


\section{Zeitlicher Verlanf der ditonischen Zncknngen.}

Um zu untersuchen, ob und wie eventuell der zeitliche Verlauf der Zuckung durch die Spannungsvermehrung beeinflusst wird, war es nöthig, monotonische und ditonische Zuckungen mit Zeitschreibung zu verzeichnen. Die Curven selbst gebe ich, da sie der wechselnden Trommelgeschwindigkeit wegen doch nicht leicht ohne weiteres mit einauder verglichen werden können, nicht wieder, dagegen sollen die folgenden Tabellen, in denen die Zuckungsdauer isotonischer und ditonischer Curven in Secunden angegeben ist, die Resultate veranschaulichen. Bei den Versuchen war die Einstellung des unteren Hebels meist eine ziemlich tiefe, so dass die zweite Last etwa im ersten Drittel der Verkürzung gehoben wurde.

Mit $m z$ ist die Dauer der gesammten monotonischen Zuckung, mit $d z$ die der ditonischen bezeichnet. Maximum $m$ bedeutet die Zeit nach Beginn der Erhebung, in welche das Maximum der monotonischen, Maximum $d$ die Zeit, in welche das Maximum der ditonischen Zuckung fiel. $p_{1}$ war stets $=7 \mathrm{~g}, p_{2}=50 \mathrm{~g}$.

\begin{tabular}{r|c|c|c|c}
\hline & mz" & $d z$ & Max. $m$ & Max. $d$ \\
\hline II. & $0,125^{\prime \prime}$ & $0,195^{\prime \prime}$ & $0,05^{\prime \prime}$ & $0,085^{\prime \prime}$ \\
II. & $0,134^{\prime \prime}$ & $0,232^{\prime \prime}$ & $0,080^{\prime \prime}$ & $0,105^{\prime \prime}$ \\
III. & $0,132^{\prime \prime}$ & $0,200^{\prime \prime}$ & $0,080^{\prime \prime}$ & $0,098^{\prime \prime}$ \\
IV. & $0,138^{\prime \prime}$ & $0,195^{\prime \prime}$ & $0,061^{\prime \prime}$ & $0,069^{\prime \prime}$ \\
V. & $0,123^{\prime \prime}$ & $0,113^{\prime \prime}$ & $0,1006^{\prime \prime}$ & $0,078^{\prime \prime}$ \\
VII. & $0,117^{\prime \prime}$ & $0,114^{\prime \prime}$ & $0,0706^{\prime \prime}$ & $0,0727^{\prime \prime}$ \\
VII. & $0,1022^{\prime \prime}$ & $0,1022^{\prime \prime}$ & $0,0613^{\prime \prime}$ & $0,0704^{\prime \prime}$ \\
VIII. & $0,1027^{\prime \prime}$ & $0,1129^{\prime \prime}$ & $0,0525^{\prime \prime}$ & $0,0672^{\prime \prime}$ \\
IX. & $0,1171^{\prime \prime}$ & $0,1655^{\prime \prime}$ & $0,0570^{\prime \prime}$ & $0,0960^{\prime \prime}$ \\
X. & $0,1358^{\prime \prime}$ & $0,842^{\prime \prime}$ & $0,0679^{\prime \prime}$ & $0,0694^{\prime \prime}$
\end{tabular}

Mit Ausnahme von V und VI zeigen die Versuche, dass die Dauer. der ditonischen Zuckung länger als die der monotoniseben ist und auch die Maximabeiditonischer Zuckung später fallen. Zur Veranschaulichung dieser Verhältnisse gebe ich in Fig. 2 ein Schema des Verhaltens ditonischer und monotonischer Zuckung. Dasselbe ist nach Nr. II der vorstehenden Tabelle entworfen, wobei ${ }^{1 / 4} \mathrm{~mm}$ der Abscisse gleich $1 / 1000^{\prime \prime}$ gesetzt wurde. Es ist wahrscheinlich, dass man mit Variirung der 
Ueb. d. Einfluss d. Spannungszunahme während d. Zuckung a. d. Arbeitsl, etc. 611

Einstellung ähnliche Resultate mit Bezug auf das Verhalten der Mäxima erhielte, wie Sogalla ${ }^{1}$ ) bei seinen Schleuderzuckungen, indem er die Verspätung der Maxima einmal von der Höhe der Einstellung der. Schleudermassen, andrerseits von dem Trägheitsmomente derselben abhängig fand. Bei. weiterer Verfolgung dieser Verhältnisse bätte man ferner den Einfluss der Temperatur auf den Zuckungsverlauf ditonischer und monotonischer Zuckungen zu berücksichtigen, wie das von Schenck für die Ansehlags- und Schleuderzuckungen unternommen wurde.

Das Wesentliche in den obigen Versuchen ist das Verhalten der Arbeitsleistungen des Muskels bei mono- und ditonischer Zuckung, sowohl nach maximaler als auch nach untermaximaler und minimaler Reizung. Ein genaueres, Verständnis aber für die Wirkung der Spannungsvermehrung während der Zuckung auf die der Arbeits-

Fig. 2.

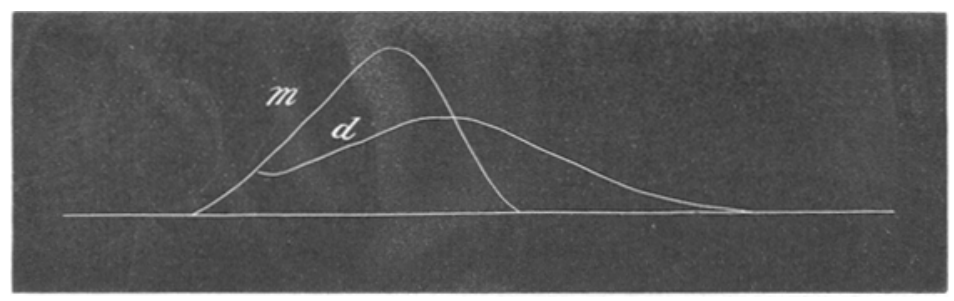

leistung zu Grunde liegenden chemischen Processe kann man erst durch myothermische Untersuchungen unter den mechanischen Bedingungen der Ditonie gewinnen. Weiterhin wäre es von Interesse, auch die elektrischen Erscheinungen am Muskel unter denselben Verhältnissen zu untersuchen.

Zum Schlusse erlaube ich mir, Professor Bernstein für die Anregung zu der Arbeit und die reichliche Hülfe, die er mir während derselben hat zu Theil werden lassen, herzlichen Dank zu sagen.

1) Sogalla, Zur mechanischen Analyse der Muskelzuckungscurven. Würzburg 1889. 


\section{Erklärung der Curven.}

[Im Interesse der Raumersparniss wurden die vom oberen und unteren Hebel gezeichneten Abscissen einander etwas näher gebracht als im Original.]

Tafel VI, Curvenfolge 1. Monotonische und ditonische Zuckungen nach maximaler Reizung. $m$ monoton. Zuckung. $d_{1} d_{2}$ ditonische Zuckungen Die verticalen Striche vor jeder Curve geben die Stellung der beiden Hebel an. Die Curven sind bei einer Trommelrotation $u=10^{\prime \prime}$ gezeichnet.

Tafel VII, Curvenfolge 2a. Monotonische und ditoniche Zackungscurven, gezeichnet bei Trommelrotation $u=1^{\prime \prime}$. Bezeichnung wie bei 1 .

Tafel VII, Curvenfolge 2b. Ditonische Zuckungen mit verschieden hoher Einstellung des Umschlages. Bezeichnung wie bei 1 und $2 \mathrm{a}$.

Tafel VI, Curvenfolge 3 a. Verkürzungs- und Spannungscurven monotonischer und ditonischer Zuckungen, auf der unteren Abscisse die Verkürzungscurven, auf der oberen, nach unten gezeichnet, die Spannungscurven (bei langsamer Trommelrotation $u=10^{\prime \prime}$ ). $m$ monotonische Zuckung. $d_{1}, d_{2}$, ditonische Zuckung.

Tafel VII, Curve 3b. Spannungs- und Verkürzungscurven einer ditonischen Zuckung; gezeichnet bei rascher Trommelrotation $\left(u=1^{\prime \prime}\right)$. 
Tabelle I. Jnseln.

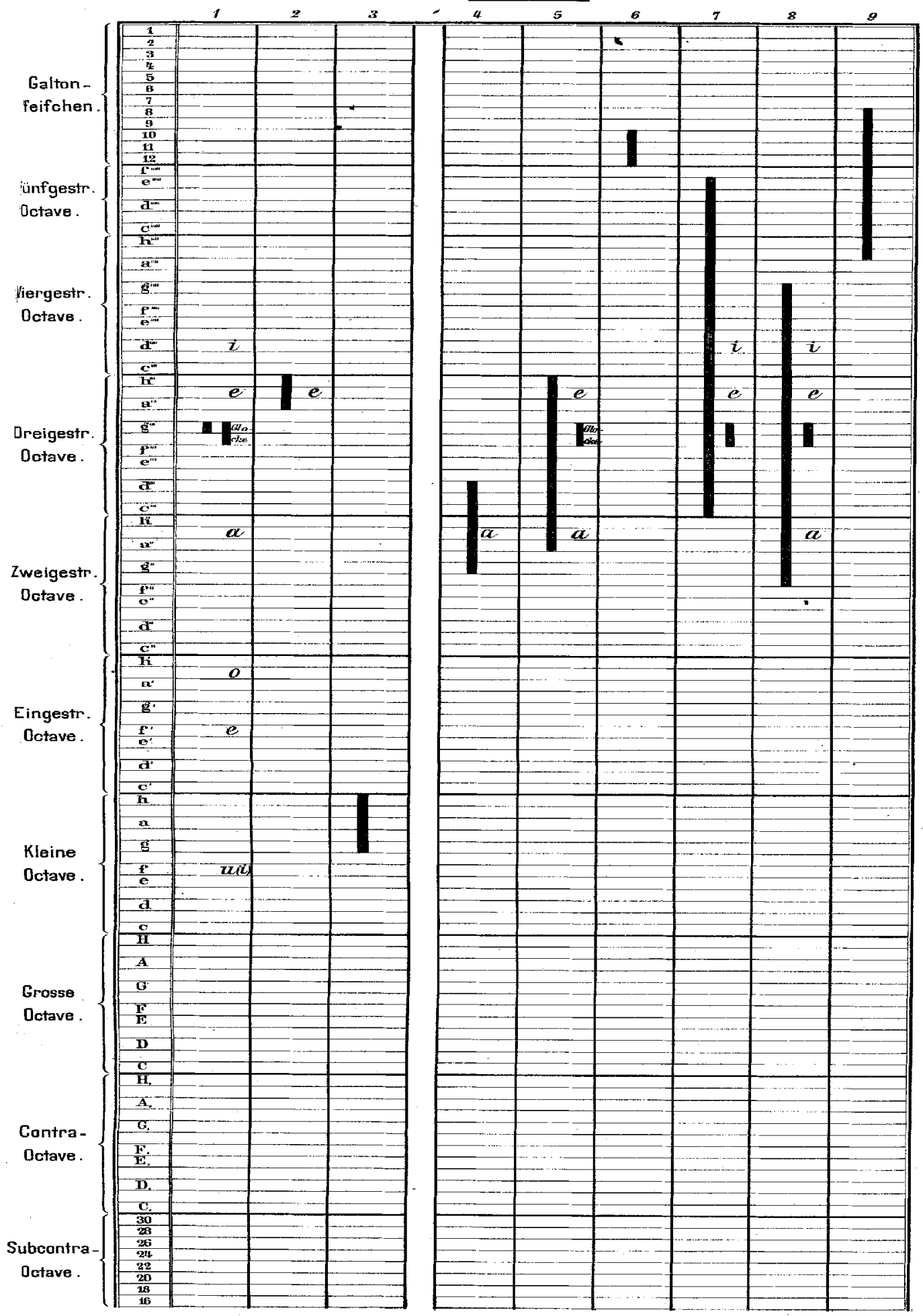

